\title{
ON-LINE GIS ANALYSIS AND IMAGE PROCESSING FOR GEOPORTAL KIELCE/POLAND DEVELOPMENT
}

\author{
B. Hejmanowska ${ }^{\mathrm{a} *}$, ${ }^{\mathrm{b}}$, E. Głowienka ${ }^{\mathrm{b}}$, R. Florek-Paszkowski ${ }^{\mathrm{b}}$ \\ ${ }^{a}$ AGH - University of Science and Technology, al. Mickiewicza 30, 30-059 Kraków, Poland, galia@agh.edu.pl \\ ${ }^{\mathrm{b}}$ Kielce University of Technology, al. Tysiąclecia Państwa Polskiego 7, 25-314 Kielce, Poland, eglo@interia.pl, rflorek@tu.kielce.pl
}

Commission II, WG II/3

KEY WORDS: GIS, Geoportal, Sentinel-2, Landsat-8, WorldView-2, MCE, OWA

\begin{abstract}
:
GIS databases are widely available on the Internet, but mainly for visualization with limited functionality; very simple queries are possible i.e. attribute query, coordinate readout, line and area measurements or pathfinder. A little more complex analysis (i.e. buffering or intersection) are rare offered. Paper aims at the concept of Geoportal functionality development in the field of GIS analysis. Multi-Criteria Evaluation (MCE) is planned to be implemented in web application. OGC Service is used for data acquisition from the server and results visualization. Advanced GIS analysis is planned in PostGIS and Python programming. In the paper an example of MCE analysis basing on Geoportal Kielce is presented. Other field where Geoportal can be developed is implementation of processing new available satellite images free of charge (Sentinel-2, Landsat 8, ASTER, WV-2). Now we are witnessing a revolution in access to the satellite imagery without charge. This should result in an increase of interest in the use of these data in various fields by a larger number of users, not necessarily specialists in remote sensing. Therefore, it seems reasonable to expand the functionality of Internet's tools for data processing by non-specialists, by automating data collection and prepared predefined analysis.
\end{abstract}

\section{INTRODUCTION}

Idea of Geoportal Kielce development was the main inspiration of the paper, as functionality of Geoportal should not be limited to the data visualization. Huge amount of the data are gathered in Geoportals and it seems that now there is time to give the end-users some help in the data analysis. Generally GIS is understood as a tool for decision support therefore also Geoportal should have this functionality. As an example, one can specify the use of spatial data in planning a new location or a new road. Based on GIS tools, one can prepare a variety of options taking into account the interests of different groups (administration, citizens, investors, greens etc.). Advanced analyses allows the criteria compensation and risk optimization. Such analyzes are available in GIS software, so why not to implement them on the Internet in Geoportals?

Other direction of Geoportal Kielce development is involving remote sensing data in urban area management (e.g. in vegetation monitoring). City vegetation is exposed to stress mainly related to air pollution and lack of water. Currently available satellite images give the possibility of widespread monitoring of urban areas if the appropriate software is available.

\section{DATA}

Existing data in Geoportal Kielce and available satellite images free of charge are shortly presented and in the chapter.

\subsection{Geoportal Kielce}

Kielce is a medium city (ca. $110 \mathrm{sq} \mathrm{km}$ ) located in south central Poland with inhabitants ca. 200000 . Since 2006 GIS in the city has been implemented and now about 120 feature classes are grouped in layers: transport, cadastre, service, investment areas, spatial planning, ownership structure, environment, culture, tourism, public services, elections, sport and orthophotomaps (http://www.gis.kielce.eu/Geoportal_toolkit/map.php).

Geoportal has predefined profiles: general, inhabitants, development and investment, spatial planning and nature and environmental protection. In addition to the visualization of these layers Geoportal Kielce has a geo-spatial analysis functionality like: search for objects, pathway selection, search for polling place, simple measurements (distance, area) and navigation (for given $\mathrm{x}, \mathrm{y}$ ). Layers are available using Web Map Service (WMS). Similar Geoportal can be found in one of the twin city: Orange in France (http://www.villeorange.fr/sortir01.htm) but with less functionality then Geoportal Kielce.

As long as one can find portals allowing the acquisition and processing of satellite images as much functionality of GIS analysis is not available. Usually basic functionality is available and some small improvements are planned (e.g. buffering WSDOT Geoportal, 2015).

Concerning satellite image processing following projects can be mentioned: forest monitoring - (EU-FP7 ZAPÁS PROJECT), cloud-based implementations, algorithms performing an atmospheric correction, land use classification and extraction of Leaf Area Index (LAI) for vegetated areas, Siberian forest monitoring (Eberle et al. 2013, 2014), project APPS4GMES operational system for Landsat data for all Germany, MELODIES Project - "technical platform", which applies cloud computing etc. The mentioned platforms are relatively

* Corresponding author 
new so it is not now possible to give any statistics about the long-live application. But on the other hand the old platforms delivering source remote sensing data (Landsat, SPOT, Hyperion USGS service etc.) are always used very intensively by wide audience. Time series predefined analyses (i.e. air pollution monitoring) are already permanently presented in Internet in EU (Copernicus, previously GMES platform) and US (Environmental Protection Agency EPA).

\subsection{Satellite imagery free of charge}

Following satellite data were analyzed:

- Landsat-8 (11 bands, pixel size: 15m - 100m),

- Sentinel-2 (13 bands, pixel size: $10 \mathrm{~m}-60 \mathrm{~m})$,

- ASTER (14 bands, pixel size: $15 \mathrm{~m}$ - 90m),

- WorldView-2 (8 bands, pixel size: 0,5m - 1,6m).

All images are download free of charge from geodatabases available from USGS (Landsat-8), NASA (ASTER), ESA (Sentinel-2, WorldView-2). Especially attention should be paid on Sentinel mission, which follows up missions: ERS, ENVISAT, SPOT, Landsat, ASTER (ESA, 2015). Comparison of the images of different spectral and spatial resolution registered in the same time is a rich source of information, particularly for environmental agency. Archive ASTER (Advanced Spaceborne Thermal Emission and Reflection Radiometer) images registered since 1999 just provided by NASA (April 2016) are an extremely valuable source of information about the land use / land cover changes. ASTER sensors register images in visible and infrared channel similar to the Sentinel-2 (Fig.1). It creates a unique opportunity to carry out multitemporal research concerning e.g. vegetation (Youhao at al. 2007; Nori \& Csaplovics, 2013), soil (Vrieling et al. 2008) or urbanisation (Xian et al. 2008; Liu\& Zhang, 2011). WorldView-2 (WV-2) just also provided free of charge in April 2016 by ESA for some EU cities are complementary to the other data. Available WV-2 data covers mainly dense urban area like in Urban Atlas. Available images WV-2 with spatial resolution $1.6 \mathrm{~m}$ ( $8 \mathrm{MS}$ bands) and $40 \mathrm{~cm}$ (1 PAN band) were collected in period: February 2011 - October 2013. Access to the data is possible through web applications: EarthExplorer, GloVis or dedicated applications, i.e. SNAP - Sentineltoolbox.

The Sentinel Toolboxes and full source code is distributed freely under the GNU GPL license.
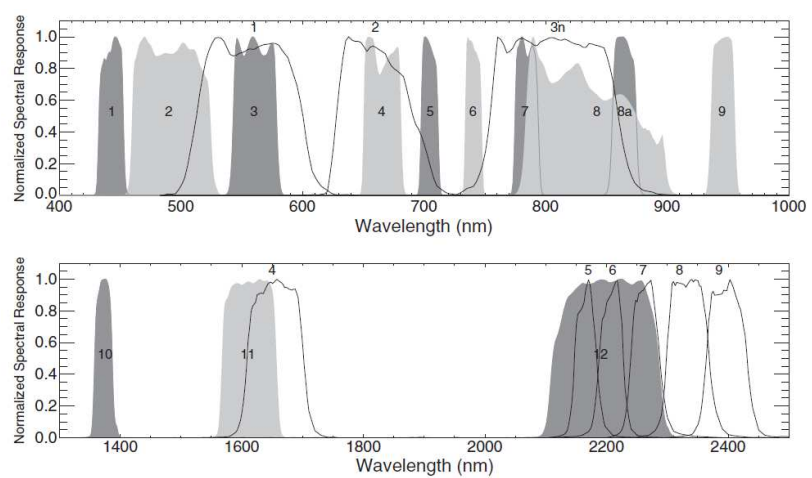

Figure 1. Spectral response functions of the Sentinel-2 (shaded) and ASTER (line) sensors, in the VNIR (top) and SWIR (bottom) wavelength range (Van der Meer et al. 2014).

\section{METHODS}

Multi-Criteria Evaluation (MCE) method is applied in advanced GIS analysis. MCE can be performed basing hard and soft method. Hard method define sharp criterions ( 0 or 1$)$ and results is obtained from simple intersection of all suitability maps corresponding to each criterion. In fuzzy logic criterions are defined as a factor describing level of suitability (i.e. value from 0 to 1). Besides suitability map for each criterion can be weighted. Additionally in Ordered Weighting Analysis (OWA) method some risk trade-off is possible by compensating of the small weight by high suitability value. MCE according the sharp criterions definition can be made both in vector and raster models. In vector model usually buffering and intersection is applied. In raster model soft criterions definition is possible. The schema of the fuzzy MCE analysis is usually as following: selection the vector layer, conversion to raster model, distance calculation, criterion definition, standardization, weighting, optional OWA, merging the criterions, constrains and final decision. In the paper some example of a new location is presented. The following criterions are defined: the near water the better, the near urban the better, the near forest the better. The constrain are assumed as following: no water, no urban and no forest. Two variants are presented, assuming equal weights for each criterion and different one. Implementation of the analysis in Geoportal needs some middleware software preparation (Fig.2). Similar approach concerning middleware can be found (Eberle et al. 2013). Vector data gathered in Geoportal are converted to raster model (V/R conversion), reclassified into $0 / 1$ map, distance from the feature is calculated, next standardization and weighting is needed, finally the criterions are merged. Last step is WMS presentation on Geoportal. Analysis are prepared in advanced PostGIS functionality (Racine, 2015). Data exchange between Geoportal, middleware and client web portal basis on the OGC service.

Second functionality development of Geoportal is presented on the right side of Fig. 2. Free of charge satellite data (Landsat 8, Sentinel 2 and new available (ASTER and WV-2) are planned to be searched periodically for Geoportal area. Data are download by wget and processed in Python (pre-processing, image classifications, indices, temperature classification etc.). Some test using SNAP, ENVI and QGIS Python Plugin: SemiAutomatic Classification written by Luca Congedo (Congedo et al. 2013) are performed. Very good results obtained using

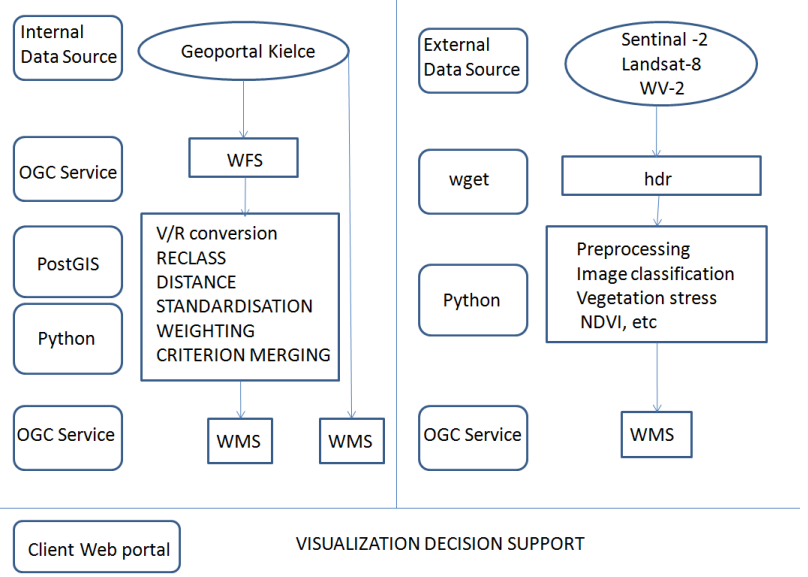

Figure. 2 Schema of Geoportal Kielce development

S-AC Plugin (speed\& quality). On the base of MTL metadata file Landsat 8 images were automatic transformed to reflectance, atmospheric corrected with DOS algorithm, transformed to the temperature and pansharpened. Images quality was improved using Minimum Noise Fraction (MNF) transformation (Green et al. 1988). Then images were classified 
using unsupervised and supervised methods: ISODATA, Maximum Likelihood (ML) and Spectral Angle Mapping (SAM) (Kruse et al. 1993).

\section{RESULTS}

MCE was performed using following layers: water, forest and urban. In postGIS layers are converted to the raster model, ST_CreateIndexRaster(), then are reclassify, ST_Reclass() and finally distance is calculated ST_EuclideanDistance(). In the next step standardization, weighting and criterion merging are programming in Python (Fig. 3). Result of advanced GIS analysis is presented on Fig. 4; water is in blue, forest in green, urban in magenta. New location with equal weights is shown in red and with different weights: water: 0.4 , forest 0.4 and urban: 0.2 in light red colour

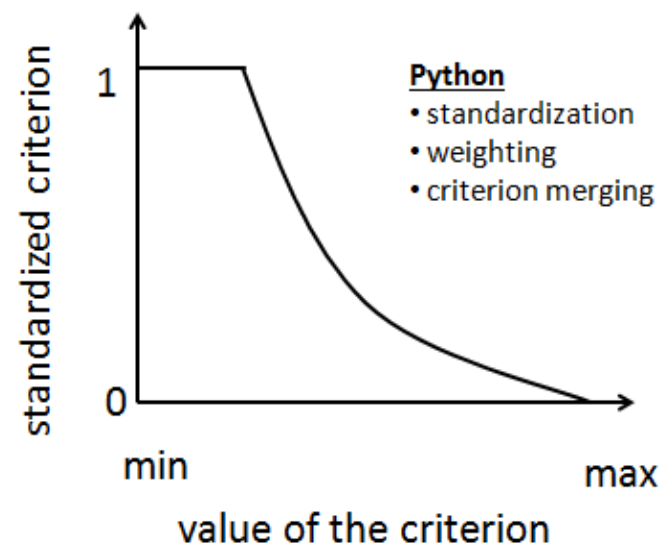

Figure.3 An example of criterion processing in Python

Application of satellites images for Geoportal Kielce is presented basing on the Sentinel-2 and Landsat-8:

- Sentinel-2: 07.08.2015, 24.03.2016

- Landsat-8: 06.08.2015, 02.04.2016

Date of the image registration is almost the same in summer 2015 and in early spring 2016.

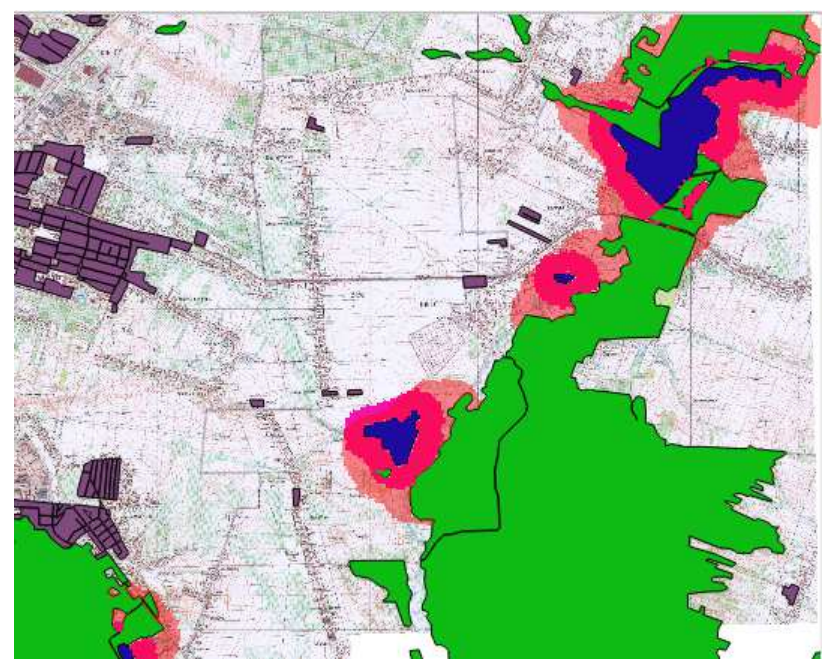

Figure.4 An example of advanced GIS analysis; MCE site location

RGB composition of Sentinale-2 is in Fig. 5 a) presented, and additionally: NDVI and LAI. Landsat- 8 images are shown in Fig. 5 b), besides RGB and NDVI temperature is in this case also presented. Results of more advanced image processing are in Fig. 5 c): MNF234 colour composite, ISODATA classification and Class Rule of SAM classification.

$$
\text { a) }
$$

b)

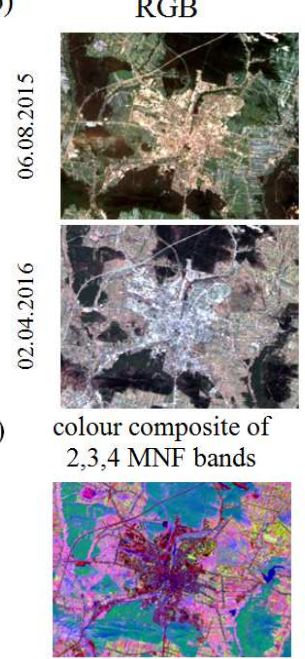

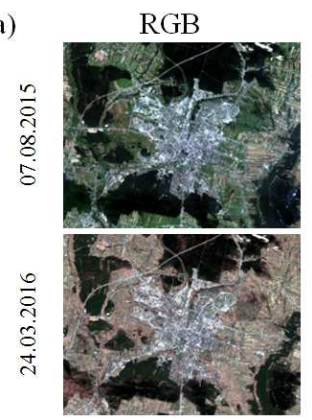

NDVI

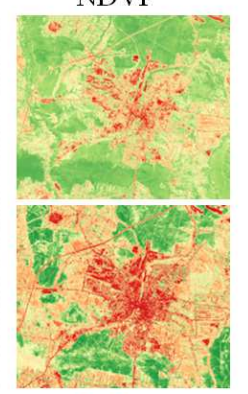

NDVI

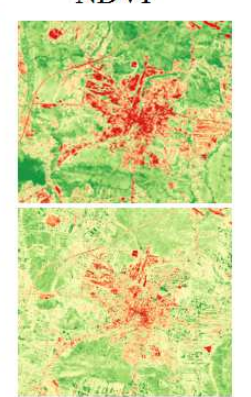

ISODATA

classification

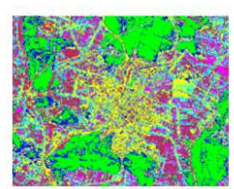

LAI

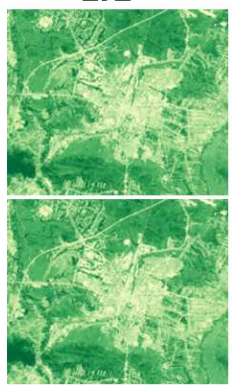

Temperature $\left[{ }^{\circ} \mathrm{C}\right]$

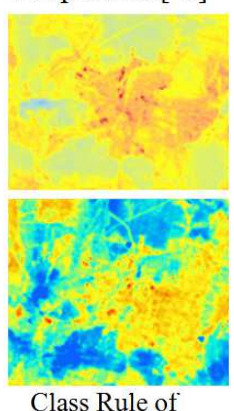

Class Rule of SAM classification

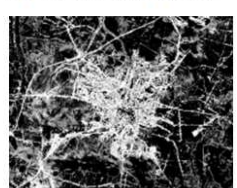

Figure 5. Images of a) Sentinel-2 b) Landsat-8 and c) their products

\section{CONCLUSIONS}

In the paper two directions of Geoportal Kielce development is presented. One concerns advanced spatial analysis which are common available in GIS software. Novelty of the approach is based on the web solution, as the functionality is available for non specialist without the necessity of using GIS software even open source. No expertise in GIS is required, criterion definition is intuitive. The same solution is proposed for image searching, downloading and processing. Predefined products are planned: colour composition (RGB, False Colour Composite), indices (e.g. NDVI), temperature, automatic image classification. Future research are planned to implement the concept in Geoportal Kielce.

\section{ACKNOWLEDGEMENTS}

Research presented in the paper is supported by AGH research no 11.11.150.949 and research of Department of Geomatics Kielce Univeristy of Technology.

\section{REFERENCES}

Congedo, L., Michele, M., Silvia, M., 2013. "Investigating the Relationship between Land Cover and Vulnerability to Climate Change in Dar es Salaam". Working Paper, Rome: Sapienza University. Available 
http://www.planning4adaptation.eu/Docs/papers/08_NWP-

DoM_for_LCC_in_Dar_using_Landsat_Imagery.pdf .

Eberle, J., Clausnitzer, S., Hüttich, C., Schmullius, C., 2013.

Multi-Source Data Processing Middleware for Land Monitoring Within a Web-Based Spatial Data Infrastructure for Siberia. ISPRS International Journal of Geo-Information, 2, 553-576.

Eberle, J., Hüttich, C., Schmullius, C., 2014. Operational Earth Observation Data Access for Automated Time-Series Monitoring based on OGC Web Processing Services. Proceedings of the ESA Big Data From Space conference 2014.

Green, A., Berman M., Switzer, B., Craig, M., 1988, A transformation for ordering multispectral data in terms of image quality with implications for noise removal. IEEE Transactions on Geoscience and Remote Sensing, 26 (1), pp. 65-74.

Kruse, F., Lefkoff, A., Boardman, J., Heidebrecht, K., Shapiro, A., Barloon, P., Goetz, A., 1993. The Spectral Image Processing System (SIPS) - Interactive Visualization and Analysis of Imaging Spectrometer Data. Remote Sensing of Environment, Special issue on AVIRIS, May-June 1993, vol. 44, pp. 145-163.

Liu, L., \& Zhang, Y. (2011). Urban heat island analysis using the Landsat TM data and ASTER data: A case study in Hong Kong. Remote Sensing, 3(7), pp.1535-1552.

Nori, W., Csaplovics, E., 2013. Integrated analysis of Aster and Landsat data to map land cover change using vegetation indices. Proceedings of the SPIE, Volume 8887, id. 8887096 pp.

Racine, P., 2015. Advanced spatial analysis with PostGIS, FOSS4G, North America, San Francisco, Hyatt San Francisco Airport, March 9 - 12, 2015

van der Meer, F.D. , van der Werff H.M.A., van Ruitenbeek F.J.A., 2014. Potential of ESA's Sentinel-2 for geological applications. Remote Sensing of Environment 148 (2014) pp. 124-133.

Vrieling, A., De Jong, S. M., Sterk, G., Rodrigues, S. C., 2008. Timing of erosion and satellite data: a multi-resolution approach to soil erosion risk mapping. International Journal of Applied Earth Observation and Geoinformation, 10(3), pp. 267-281.

Xian, G., Crane, M., McMahon, C., 2008. Quantifying multitemporal urban development characteristics in Las Vegas from Landsat and ASTER data. Photogrammetric Engineering \& Remote Sensing, 74(4), pp. 473-481.

Youhao, E. Wang, J., Gao, S., Yan, P., Yang, Z., 2007. Monitoring of Vegetation Changes Using Multi-temporal NDVI in Peripheral Regions around Minqin Oasis, Northwest China. Geoscience and Remote Sensing Symposium, 2007. IEEE International, pp. 3448-3451.

Sentinel Toolboxes, 2015. https://sentinel.esa.int/web/sentinel/toolboxes.

WSDOT Geoportal, 2015. Washington State Department of Transportation Geoportal, http://www.wsdot.wa.gov/mapsdata/tools/Geoportal_ext.htm 